\title{
AVIAN MALARIA SEROPREVALENCE IN JACKASS PENGUINS (SPHENISCUS DEMERSUS) IN SOUTH AFRICA
}

\author{
Thaddeus K. Graczyk, Jean J. Brossy*, Andrea Plösł, and Michael K. Stoskopf $\ddagger$ \\ The Johns Hopkins University, School of Hygiene and Public Health. \\ Department of Molecular Microbiology and Immunology, 615 North Wolfe Street, Baltimore, Maryland 21205
}

\begin{abstract}
Blood samples of 191 adult Jackass penguins (Spheniscus demersus) from South Africa were tested by indirect enzyme-linked immunosorbent assays (ELISA) with Plasmodium falciparum antigen (R32tet ${ }_{32}$ ) for avian malaria antibodies (Ab). The samples originated from free-ranging penguins from offshore islands and southern coast colonies $(3 \mathrm{groups}, \mathrm{n}=110)$, from 2 penguin groups $(n=66)$ rescued after offshore oil-spill contamination and rehabilitated at the Rescue Station of the South African National Foundation for the Conservation of Coastal Birds (SANCCOB) in Cape Town, and from SANCCOB-resident penguins $(n=15)$. The total average malaria Ab seroprevalence was $39 \%$, and the mean malaria seropositivity ranged from $20 \%$ to $62 \%$ among the $6 S$. demersus groups, with a mean of $55 \%$ for oiled penguins, and $31 \%$ for the remaining birds. The total mean absorbance value was 0.57 for ELISA-positive penguins, 0.43 for birds kept at SANCCOB facilities, and 0.70 for the penguins from wild colonies. The 2 groups of oiled penguins exhibited higher malaria Ab seroprevalence (38\% and $62 \%)$ than the 3 groups of non-oiled birds ( $29 \%, 33 \%$ and $35 \%$ ). Malaria Ab seroprevalence of free-ranging penguins was significantly lower $(P<0.01)$ than in the 3 groups of birds at SANCCOB facilities and did not differ significantly among 3 wild penguin colonies.
\end{abstract}

The Jackass, or African black-footed penguin Spheniscus demersus is an endangered species, with decreasing populations along the southern coast and offshore islands of South Africa (SA) (Crawford et al., 1990) due to oil-spill contaminations (Holmes, 1973), injuries, diseases (Brossy, 1992), decline of prey populations, and environment degradation (Stoskopf, 1993). Plasmodium relictum-associated avian malaria is a high-mortality disease of captive penguins in North America (Stoskopf and Beier, 1979; Cranfield et al., 1990) and a recognized disease of wild and stranded Jackass penguins (Brossy, 1992). The earliest penguin malarias ( $P$. relictum) with parasitemia prevalences of $5 \%$ and $7 \%$ were found in 2 separate collections of wild $S$. demersus from Saldanha Bay $\left(32^{\circ} 26^{\prime} \mathrm{S}, 17^{\circ} 455^{\prime} \mathrm{E}\right)$ (SA) in 1927 (Fantham and Porter, 1944). Two percent of the vector mosquitoes, Culex quinquefasciatus (referred to as Culex fatigans in that study), had oocysts in the midgut, and $1 \%$ of the mosquitoes had salivary gland sporozoites (Fantham and Porter, 1944). Over 60 yr later, Brossy (1992) reported a $15 \%$ prevalence of $P$. relictum in injured or oiled wild $S$. demersus rescued along the southern SA coast and rehabilitated at the Rescue Station of the South African National Foundation for the Conservation of Coastal Birds (SANCCOB) in Milnerton, Cape Town $\left(33^{\circ} 55^{\prime} \mathrm{S}\right.$, $\left.18^{\circ} 22^{\prime} \mathrm{E}\right)$.

Brossy (1992) found only 1 putatively positive $P$. relictum identification in 140 wild $S$. demersus from offshore $S A$ islands and concluded that malaria does not normally occur in wild penguins, but endemic malaria could be transmitted to them after capture and confinement in restricted areas. If so, SANCCOB-released birds exposed to parasites in captivity could represent a potential malaria hazard for the wild penguins in areas where Culex spp. mosquitoes are present.

Received 8 March 1995; revised 28 April 1995; accepted 28 April 1995.

* University of Cape Town, Medical School Observatory 7925, Department of Anatomy and Cell Biology, Cape Town, South Africa.

+ Percy FitzPatrick Institute of African Ornithology, University of Cape Town, Rondebosch 7700, South Africa.

$\ddagger$ North Carolina State University, College of Veterinary Medicine, Department of Companion Animal and Special Species Medicine, 4700 Hillsborough Street, Raleigh, North Carolina 27606.
Parasitemia prevalence may not reflect the actual extent of infection in a penguin population. Recrudescence and relapses of malarias have been reported in many avian species (Atkinson and Van Riper, 1991), including captive Jackass penguins (Stoskopf and Beier, 1979; Cranfield et al., 1994) and only 1 of 38 wild-caught Magellanic penguins (Spheniscus magellanicus) that died because malaria in 1 outbreak was parasitemic (Fix et al., 1988).

The enzyme-linked immunosorbent assay (ELISA) for the diagnosis of $P$. relictum or Plasmodium elongatum infections in captive $S$. demersus can determine the exposure of the penguins to the parasites (Graczyk, Cranfield, Skjoldager, and Shaw, 1994). The purpose of this study was to compare the seroprevalence of malarial antibodies (Ab) in wild free-ranging Jackass penguins from offshore islands and southern SA coast with those of rescued penguins after offshore oil-spill contamination and held at SANCCOB for rehabilitation.

\section{MATERIALS AND METHODS}

A total of 191 blood samples of adult $S$. demersus from SA collected from December 1993 to July 1994 according to the protocol of Earlé et al. (1993) was used. The samples originated from penguin colonies on Dassen Island, Robben Island, and Boulders located on the mainland (Table I). The collection includes samples from Dassen Island penguins rescued after offshore oil-spill contamination and rehabilitated for at least 2 wk at SANCCOB and samples from the resident SANCCOB penguin population (Table I).

The blood samples were air-dried and stored on filter paper as described by Graczyk et al. (1993). Storage on filter paper does not diminish Ab binding capacity (Graczyk et al., 1993). The blood samples were eluted into buffer (Graczyk et al., 1993), and the presence of antiPlasmodium spp. immunoglobulins was determined by indirect ELISA (Graczyk, Cranfield, Skjoldager, and Shaw, 1994) with Plasmodium falciparum sporozoite R32tet ${ }_{32}$ antigen $(2 \mu \mathrm{g} / \mathrm{ml})$. Immunoglobulins against $P$. relictum and $P$. elongatum recognize this antigen (Graczyk et al., 1993) but do not cross-react with the antigens of other avian blood hacmosporidians (Graczyk, Cranfield, and Shiff, 1994). The elute and alkaline phosphatase-labeled rabbit anti-S. demersus $\operatorname{lgG}$ were used at dilutions of $1 / 100$ and $1 / 1000$, respectively. Eight wells on each ELISA plate were not coated with R32tet ${ }_{12}$ antigen to determine nonspecific background absorbance values. The positive control (PC) was a serum pool of 5, 2-yr-old captive $S$. demersus, clinically infected with $P$. relictum (2 penguins) and $P$. elongatum confirmed by the blood subinoculation method of Herman et al. (1966). These samples were selected because they had the highest absorbances reported during our 1 -yr-ELISA-monitoring period of captive $S$. demersus. Pooled plasma 
TABLE I. Seroprevalence for anti-Plasmodium spp. immunoglobulins in adult Jackass penguins (Spheniscus demersus) rehabilitated after oil spill contamination at the Rescue Station of the South African National Foundation for the Conservation of Coastal Birds (SANCCOB) in Cape Town, South Africa, and in free-ranging penguins from wild colonies determined by enzyme-linked immunosorbent assay (ELISA) utilizing $P$. falciparum sporozoite (R32tet ${ }_{32}$ ) antigen.

\begin{tabular}{|c|c|c|c|c|c|}
\hline \multirow[b]{3}{*}{ Geographical location of penguins } & \multicolumn{3}{|c|}{ Penguins } & \multirow{2}{*}{\multicolumn{2}{|c|}{ Absorbance }} \\
\hline & \multirow{2}{*}{$\begin{array}{c}\text { Total } \\
\text { no. }\end{array}$} & \multirow{2}{*}{$\begin{array}{c}\text { Positive* } \\
\text { no. }\end{array}$} & \multirow[b]{2}{*}{$(\%)$} & & \\
\hline & & & & Range & Mean $\pm \mathrm{SE}$ \\
\hline †SANCCOB, $33^{\circ} 55^{\prime} \mathrm{S} 18^{\circ} 22^{\prime} \mathrm{E}$ & 21 & 8 & (38) & $0.20-0.85$ & $0.38 \pm 0.02$ \\
\hline †SANCCOB, $33^{\circ} 55^{\prime} \mathrm{S} 18^{\circ} 22^{\prime} \mathrm{E}$ & 45 & 28 & (62) & $0.22-0.53$ & $0.42 \pm 0.04$ \\
\hline Robben Island, $33^{\circ} 49^{\prime} \mathrm{S} 18^{\circ} 22^{\prime} \mathrm{E}$ & 46 & 16 & (35) & $0.27-1.00$ & $0.53 \pm 0.06$ \\
\hline$\S S A N C C O B, 33^{\circ} 55^{\prime} \mathrm{S} 18^{\circ} 22^{\prime} \mathrm{E}$ & 15 & 3 & (20) & $0.40-0.54$ & $0.48 \pm 0.04$ \\
\hline Dassen Island, $33^{\circ} 26^{\prime} \mathrm{S} 18^{\circ} 04^{\prime} \mathrm{E}$ & 30 & 10 & (33) & $0.51-1.18$ & $0.80 \pm 0.07$ \\
\hline Boulders, Simons Town, $34^{\circ} 14^{\prime} \mathrm{S} 18^{\circ} 26^{\prime} \mathrm{E}$ & 34 & 10 & (29) & $0.59-1.19$ & $0.78 \pm 0.07$ \\
\hline
\end{tabular}

- Above the cutoff level of $405 \mathrm{~nm}$ wavelength absorbance (0.19) equal to the mean absorbance \pm 3 SD of 8 negative controls.

+ Penguins originated from Dassen Island and rehabilitated at SANCCOB after oil spill for at least 2 wk.

$\ddagger$ Oiled penguins.

\$ Resident penguin population.

from 4, 3-mo-old $S$. demersus juveniles, housed indoors in mosquitofree conditions (Graczyk, Cranfield, Shaw, and Craig, 1994), were used as a negative control (NC) in 8 wells per plate. At this age, maternally transmitted malarial Abs were not detectable by our ELISA (Graczyk, Cranfield, Shaw, and Craig, 1994). The malaria-negativity of the juveniles was confirmed by the blood subinoculation method (Herman et al., 1966). The indirect ELISA protocol followed our previous procedures (Graczyk, Cranfield, Skjoldager, and Shaw, 1994), and the method of Schwartz et al. (1991) was used to compare the absorbance values from different ELISA plates. The positive cutoff level for ELISA was established as an absorbance greater than the mean $\pm 3 \mathrm{SD}$ of $8 \mathrm{NC}$ wells.

Statistical analysis was performed with Analytical Software Statistix 4.1 (Analytical Software, St. Paul, Minnesota). Kruskal-Wallis analysis of variance (ANOVA) was performed to determine the significance of among-penguin group effects in absorbance values. A rank sum test was used to compare the mean absorbance between penguin groups, and the $G$-heterogeneity test (Sokal and Rohlf, 1981) was applied to compare seroprevalence among penguin groups. Statistical significance was considered to be $P<0.05$.

\section{RESULTS}

The range of absorbance for positive control was $0.61-0.98$ with a mean $\pm \mathrm{SE}$ of $0.83 \pm 0.02$. The negative control absorbance values ranged from 0.10 to 0.15 with a mean of $0.13 \pm$ 0.01 ( $\mathrm{SD}=0.02$ ), establishing a cutoff absorbance level of 0.19 . Nonspecific background absorbances did not exceed 0.07 .

The seroprevalence of malarial Ab ranged from $20 \%$ to $62 \%$ among the $S$. demersus groups (Table I), with a total mean avian malaria seropositivity of $39 \%$. The 2 oiled penguin groups had a mean seroprevalence of $55 \%$ compared to $31 \%$ for the remaining birds ( $G$-heterogeneity test; $G=6.33, P<0.02$ ). The mean seroprevalence of birds at SANCCOB facilities was $48 \%$, versus 33\% for free-ranging penguins originating from Dassen Island, Robben Island, and Boulders.

The $G$-heterogeneity test showed a significant $(G=9.82, P$ $<0.05)$ difference in the prevalence of malaria seropositivity among the 6 penguin groups. However, when the 2 groups of oiled birds were excluded from analysis, the differences among the remaining 4 groups were not significant. The seroprevalence of the groups of penguins rescued after oil spills along the southern coast of SA $(62 \%)$ was significantly higher ( $G$-heterogeneity test; $G=24.45, P<0.01)$ than the malarial seropositivity of oiled penguins originating from Dassen Island (38\%) (Table I).

The total mean absorbance value $( \pm$ SE) for all penguins was $0.57 \pm 0.03$. The mean absorbance value $( \pm S E)$ for all oiled, rescued, and rehabilitated birds held at SANCCOB was $0.43 \pm$ 0.02 . Free-ranging penguins from the colonies in Dassen Island, Robben Island, and Boulders colonies had a mean absorbance value $( \pm S E)$ of $0.70 \pm 0.04$. All these means were significantly lower (rank sum test; $t=6.39, P<0.01$ ) than the mean absorbance of captive $S$. demersus used as our positive control. Interestingly, the 2 groups of oiled penguins exhibited significantly lower (rank sum test; $t=5.01, P<0.01$ ) mean values of absorbance than the other birds (Table I) but had the highest malaria seroprevalence (Fig. 1). The absorbances varied significantly among $6 \mathrm{~S}$. demersus groups (Kruskal-Wallis ANOVA; $F=6.04, P<0.01$ ), even when the oiled penguins were excluded from testing (Kruskal-Wallis ANOVA; $F=4.85, P<0.01$ ). The mean absorbance values did not differ significantly among 3 penguin groups kept at SANCCOB (Table I).

\section{DISCUSSION}

An accurate assessment of naturally occurring exposure to avian malaria in free-ranging Jackass penguins is important in understanding the role that the disease plays in the population dynamics of this endangered species. It is also crucial to evaluate the risk of releasing (possibly infected) rehabilitated penguins to the wild. The conclusion of Brossy (1992) that avian malaria does not occur in wild Jackass penguins agrees with the observation of Fantham and Porter (1944) that parasitemia prevalence of wild penguins was considerably lower than expected considering the abundance of mosquito vector $C$. quinquefasciatus (referred to as $C$. fatigans in that study) and the social behavior of the penguin. Fantham and Porter (1944) explained this phenomenon by low gametocytemia, penguin age-related immunity to malarias, mosquito-impeding feathers, and escape into the water of penguins from mosquitoes. The last feature was also listed by Brossy (1992). However, the oil-spill contamination that immobilizes a penguin and impairs the feather bar- 


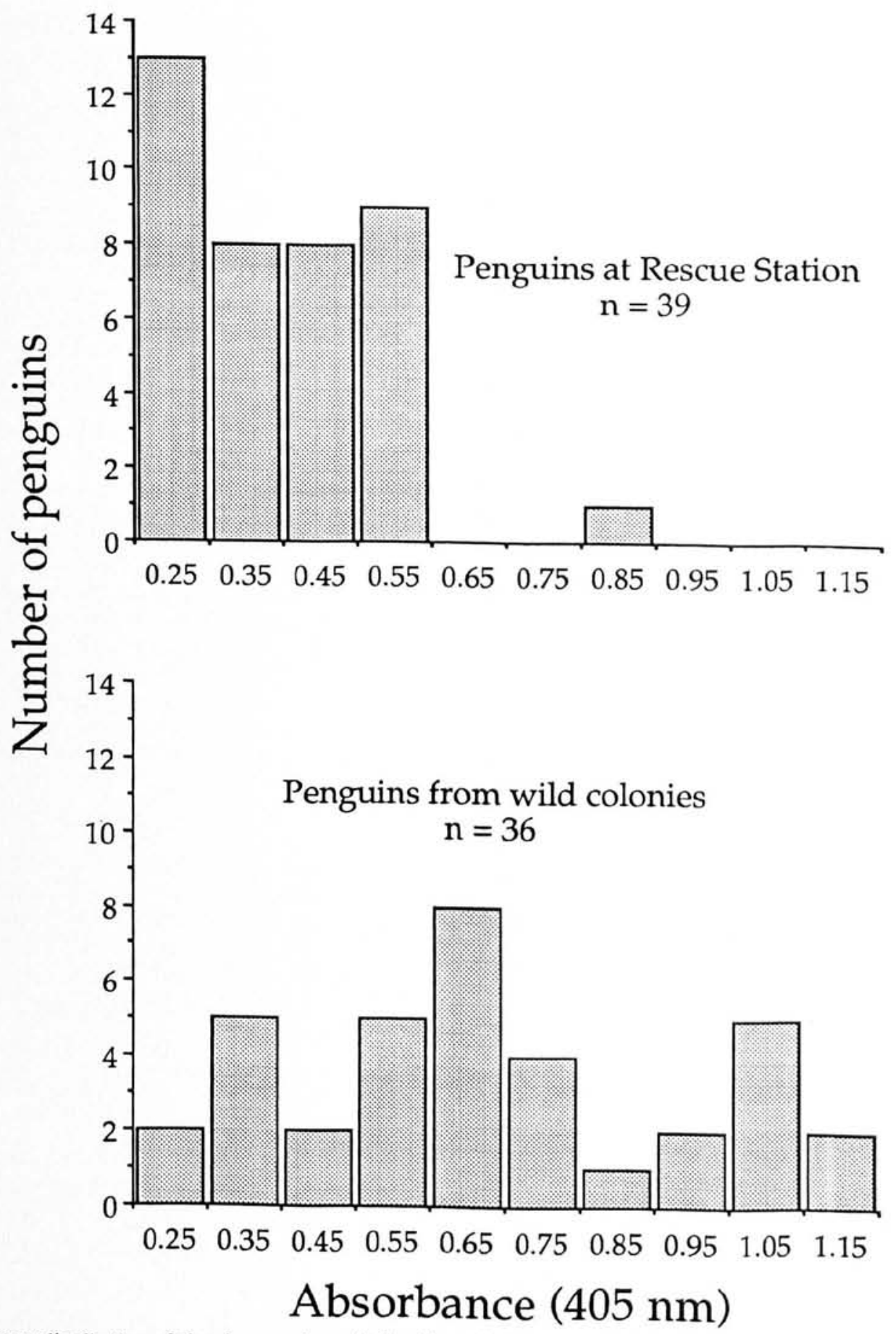

FIGURE 1. Frequency distribution of absorbance values obtained in an indirect enzyme-linked immunosorbent assay (ELISA) utilizing Plas modium falciparum sporozoite ( $\mathrm{R}_{2}$ tet $_{32}$ ) antigen for detection of anti-Plasmodium spp. immunoglobulins in adult Jackass penguins (Spheniscus demersus) rehabilitated after offshore oil-spill contamination at the Rescue Station of the South African National Foundation for the Conservation of Coastal Birds (SANCCOB) in Cape Town, South Africa, and in free-ranging penguins from wild colonies.

rier may make a bird more vulnerable to mosquito attack. Culex spp. mosquitoes have been reported from the coastal areas that serve as natural habitat of Jackass penguins (Knight and Stone, 1977).

The ELISA results indicate more intense exposure to malarial parasites in the penguins kept at the Rescue Station of SANC-
COB than in birds in the wild. An additional feature of SANCCOB-rehabilitated birds is the homogeneity in Ab titers with generally lower titers than in the penguins from wild colonies. Brossy (1992) reported that rescued wild penguins may contract malaria while being rehabilitated at SANCCOB where the parasitemia prevalence may reach $25 \%$. As mentioned (Brossy, 
1992), the released birds tend to return to their own colonies. Over $87 \%$ of Jackass penguins rehabilitated at SANCCOB after oil-spill contamination had returned to former nests and mates (Randall et al., 1980). Thus, malarial seropositivity in the penguins from wild colonies may not fully reflect the exposure to parasites in their natural habitat. Seropositivity may also be explained by maternally transmitted Ab (Graczyk, Cranfield, Shaw, and Craig, 1994); however, detection of maternal Ab was excluded in the present study because all the penguins were adult. Malarial seropositivity of penguins in the areas with no documented Culex spp. occurrence may be explained by exposure while in other locations or during migration.

It is necessary to point out that, in order to standardize the ELISA, our positive control was a pool of captive $S$. demersus sera selected due to high absorbances. The mean absorbance values of wild $S$. demersus in the present study were in the lower range of captive $S$. demersus in North America (see Graczyk, Cranfield, McCutchan, and Bicknese, 1994). However, the Ab titers of wild penguins cannot be directly compared with the $\mathrm{Ab}$ titers of captive penguins held under long-term avian malaria control programs (Stoskopf and Beall, 1980). Most of the species of wild penguins breed in areas free of malaria vectors and these penguins are naive to avian species of Plasmodium (Bennett et al., 1993). The pharmacological treatment (Cranfield et al., 1990) of captive penguins reduces fatality and consequently increases the risk of subsequent exposure to the parasites with a boost of the humoral responses.

Offshore oil-spill contamination of the body surfaces of marine birds affects thermoinsulation, thermoregulation, and rate of metabolism (Jenssen et al., 1985) and can cause systemic toxic effects if the oil is ingested (Leighton, 1985). Erasmus et al. (1981) measured the time spent in water of wild Jackass penguins that were experimentally covered by oil on $70 \%$ of the body surface. They found that oiled birds leave the water within $4 \mathrm{~min}$ of entering, whereas control penguins stayed voluntarily in the water for the length of the experiment. Similar observations were noted by Culik et al. (1991) on oil-contaminated, wild Adelie penguins (Pygoscelis adeliae). Additionally, oiled birds displayed erratic swimming behavior. Thus, the heat losses due to oil-impaired thermal properties of the feathers in Jackass penguins (Erasmus et al., 1981) may affect the penguin defensive behavior against mosquitoes of escaping into the water (Fantham and Porter, 1944; Brossy, 1992). Oiled Jackass penguins developed fatal Salmonella typhimurium and Staphylococcus aureus infections (Westphal and Rowan, 1970).

It was postulated (Stoskopf, 1993) that penguins may not respond physiologically to oil contamination in the same manner as ducks. Jenssen et al. (1985) demonstrated that the high metabolic rate observed in experimentally oil-contaminated ducks in the laboratory did not apply to field conditions. It has been shown that natural oil contamination of the body surface results in a decreased body temperature of wild Jackass penguins (Erasmus et al., 1981) and decreased metabolic rate in wild Black-billed murre (Uria aalge) (Jenssen et al., 1985). The comprehensive explanation of this phenomenon is given by Jenssen et al. (1985). Culik et al. (1991) reported that oil-contaminated wild $P$. adeliae had reduced heart rates, body temperatures, and energy expenditures when compared with control, non-oiled birds.

The oiled penguin groups in the present study displayed sig- nificantly lower $\mathrm{Ab}$ responses to malarial parasites than healthy birds in wild colonies. Studies on humoral responses in oiled birds do not exist; however, it has been shown that oil ingestion induces kidney, endocrine system, and membrane transport malfunction (Hartung and Hunt, 1966; Crocker et al., 1974), and morphologic lesions in the erythrocytes (Leighton, 1985). Oil ingestion decreases bird blood thyroxine levels and corticosterone levels (Rattner and Eastin, 1981). Oil contamination of penguin feathers has a long-term effect in Jackass penguins, and even a natural moult does not rid a bird of its oil load (Kerley et al., 1985). We conclude that the weaker Ab responses in Jackass penguins to malarial parasites rescued from offshore oil-spill contamination reflects the exposure to oil pollution.

A major concern in developing re-release policies is that penguins released from $\mathrm{SANCCOB}$ return to former nests and mates (Randall et al., 1980). Previous studies showed that chloroquine/primaquine antimalarial therapy failed to eliminate malarias from captive Jackass (Stoskopf and Beier, 1979; Cranfield et al., 1994) or wild-caught Magellanic penguins ( $S$. magellanicus) (Fix et al., 1988). It is doubtful that chloroquine/primaquine treatment may be fully effective for wild Jackass penguins either for therapy or prophylaxis. Thus, the antimalarial treatment of wild penguins at SANCCOB facilities (Brossy, 1992) may not free the birds of contracted malarias. This may represent a potential malaria hazard when birds return to their own colonies, even without the presence of mosquitoes, because of potential environmental hazard(s) that may trigger parasite recrudescence or relapse from subclinical infections.

\section{ACKNOWLEDGMENTS}

We acknowledge F. Barnes for technical assistance and $\mathrm{K}$. Wolff for editorial comments. We thank SmithKline Beecham Pharmaceuticals for providing R32tet ${ }_{32}$ antigen. This study was supported by the AKC Fund of New York.

\section{LITERATURE CITED}

Atkinson, C.T., AND C. VAN RIPER III. 1991. Pathogenicity and epizootiology of avian hematozoa: Plasmodium, Leucocytozoon, and Haemoproteus. In Bird-parasite interactions, ecology, evolution and behaviour, J. E. Loye and M. Zuk (eds.). Oxford University Press, New York, New York, p. 20-48.

Bennett, G. F., M. A. Peirce, And R. W. Ashford. 1993. Avian hematozoa: Mortality and pathogenicity. Journal of Natural History 27: 993-1001.

BRossy, J. J. 1992. Malaria in wild and captive Jackass penguins Spheniscus demersus along the southern African coast. Ostrich 63: 10-12.

Cranfield, M. R., T. K. Graczyk, F. B. Beall, D. M. Ialeggio, M. L. SHAW, AND M. L. SKJOLdAGER. 1994. Subclinical avian malaria infections in African black-footed penguins (Spheniscus demersus) and induction of parasite recrudescence. Journal of Wildlife Diseases 30: 372-376.

- , M. L. Shaw, F. B. Beall, AND M. L. SkJoldager. 1990. Review and update of avian malaria in African penguins (Spheniscus demersus). Proceedings of the American Association of Zoo Veterinarians 21: 234-248.

Crawford, R. J. M., A. J. Williams, R. M. Randall, A. Berruti, AND G. J. B. Ross. 1990. Recent population trends of Jackass penguins off Southern Africa. Biological Conservation 52: 229-243.

Crocker, A. D., J. Cronshaw, and W. N. Holmes. 1974. The effect of crude oil on intestinal absorption in ducklings (Anas platyrhynchos). Environmental Pollution 7: 165-177.

Culik, B. M., R. P. WILSON, A. T. WOAKES, AND F. W. SANudo. 1991. 
Oil pollution in Antarctic penguins: Effects on energy metabolism and physiology. Marine Pollution Bulletin 22: 388-391.

EARLÉ, R. A., F. W. HuChzermeYer, AND J. J. Brossy. 1993. Babesia peircei sp. nov. from the Jackass penguin. South African Journal of Zoology 28: 88-90.

Erasmus, T., R. P. Mason, AND G. I. H. Kerley. 1985. Effect of moult on crude oil load in a jackass penguin Spheniscus demersus. Marine Pollution Bulletin 16: 474-476.

- R. M. Randall, AND B. M. Randall. 1981. Oil pollution, insulation and body temperatures in the Jackass penguin Spheniscus demersus. Comparative Biochemistry and Physiology 69A: 169171.

Fantham, H. B., and A. Porter. 1944. On a Plasmodium (Plasmodium relictum var. spheniscidae, $\mathrm{n}$. var.), observed in four species of penguins. Proceedings of the Zoological Society of London 114: $279-292$.

Fix, A. S., C. WAterhouse, E. C. Greiner, AND M. K. Stoskopf. 1988. Plasmodium relictum as a cause of avian malaria in wild-caught Magellanic penguins (Spheniscus magellanicus). Journal of Wildlife Diseases 24: 610-619.

Graczyk, T. K., M. R. Cranfield, T. F. McCutchan, and E. J. BICKNESE. 1994. Characteristics of naturally acquired avian malaria infections in naive juvenile African black-footed penguins (Spheniscus demersus). Parasitology Research 80: 634-637.

M. L. Shaw, AND L. E. Craig. 1994. Anti-Plasmodium spp. maternal antibodies in African black-footed penguin (Spheniscus demersus) chicks. Journal of Wildlife Diseases 30: 365-371.

AND C. J. SHIFF. 1993. ELISA method for detecting anti-Plasmodium relictum and anti-Plasmodium elongatum antibody in infected duckling sera using Plasmodium falciparum antigens. Journal of Parasitology 79: 879-885.

- AND 1994. Extraction of Haemoproteus columbae (Haemosporina: Haemoproteidae) antigen from rock dove pigeons (Columba livia) and its use in antibody ELISA. Journal of Parasitology 80: 713-718.

, M. L. SkJoldager, AND M. L. Shaw. 1994. An ELISA for detecting anti-Plasmodium spp. antibodies in African blackfooted penguins (Spheniscus demersus). Journal of Parasitology 80: $60-66$.

- M. L. Shaw, M. R. Cranfield, and F. B. Beall. 1994. Hematologic characteristics of avian malaria cases in African blackfooted penguins (Spheniscus demersus) during the first outdoor exposure season. Journal of Parasitology 80: 302-308.

HARTUNG, R., AND G. S. HuNT. 1966. Toxicity of some oils to waterfowl. Journal of Wildlife Management 30: 564-570.
Herman, C. M., J. O. Kingsley, and E. A. Snyder. 1966. Subinoculation as a technique in the diagnosis of avian Plasmodium. Avian Diseases 10: 541-547.

HolmEs, M. 1973. Oil and penguins don't mix. National Geographic 150: $384-396$.

JENSSEN, B. M., M. EKKER, AND C. BECH. 1985. Thermoregulation in a naturally oil-contaminated Black-billed murre Uria aalge. Bulletin of Environmental Contamination and Toxicology 35: 9-14.

Kerley, G. I. H., T. Erasmus, and R. P. Mason. 1985. Effect of moult on crude oil load in a Jackass penguin Spheniscus demersus. Marine Pollution Bulletin 16: 474-476.

KNIGHT, K. L., AND A. StonE. 1977. A catalog of the mosquitoes of the world. The Thomas Say Foundation, Entomological Society of America, Baltimore, Maryland, 611 p.

Leighton, F. A. 1985. Morphologic lesions in red blood cells from Herring gulls and Atlantic puffins ingesting Prudhoe Bay crude oil. Veterinary Pathology 22: 393-402.

RANDALl, R. M., B. M. RANDALl, AND J. Bevan. 1980. Oil pollution and penguins-Is cleaning justified? Marine Pollution Bulletin 11: 234-237.

RATTNER, B. A., AND W. C. EASTIN. 1981. Plasma corticosterone and thyroxin concentrations during chronic ingestion of crude oil in mallard ducks (Anas platyrhynchos). Comparative Biochemistry and Physiology 68C: 103-107.

Schwartz, B. S., D. P. Ford, J. E. Childs, N. Rothman, AND R. J. ThомAs. 1991. Anti-tick saliva antibody: A biologic marker of tick exposure that is a risk factor for Lyme disease seropositivity. American Journal of Epidemiology 134: 86-95.

SoKAL, R. R., AND F. J. ROHLF. 1981. Biometry, 2nd ed. W. H. Freeman and Co., New York, New York, 859 p.

Stoskopf, M. K. 1993. Penguin and alcid medicine. In Zoo \& wild animal medicine. M. E. Fowler (ed.). W. B. Saunders Co., Division of Hartcourt Brace \& Co., Philadelphia, Pennsylvania, p. 189-194.

, AND F. B. BEALL. 1980. The husbandry and medicine of captive penguins. Proceedings of the American Associations of Zoo Veterinarians 11: $81-96$.

, AND J. R. BEIER. 1979. Avian malaria in African black-footed penguins. Journal of the American Veterinary Medical Association 175: 944-947.

WestPhal, A., AND M. K. Rowan. 1970. Some observation on the effects of oil pollution on the Jackass penguin. Ostrich 41(Suppl.): 521-526. 